\title{
Self-disseminating vaccines to suppress zoonoses
}

\author{
Scott L. Nuismer ${ }^{1,2} \llbracket$ and James J. Bull'
}

\begin{abstract}
The SARS-CoV-2 epidemic is merely the most recent demonstration that our current approach to emerging zoonotic infectious disease is ineffective. SARS, MERS, Ebola, Nipah and an array of arenavirus infections sporadically spillover into human populations and are often contained only as a result of their poor transmission in human hosts, coupled with intense public health control efforts in the early stages of an emerging epidemic. It is now more apparent than ever that we need a better and more proactive approach. One possibility is to eliminate the threat of spillover before it occurs using vaccines capable of autonomously spreading through wild animal reservoirs. We are now poised to begin developing self-disseminating vaccines targeting a wide range of human pathogens, but important decisions remain about how they can be most effectively designed and used to target pathogens with a high risk of spillover and/or emergence. In this Perspective, we first review the basic epidemiological theory establishing the feasibility and utility of self-disseminating vaccines. We then outline a road map for overcoming remaining technical challenges: identifying high-risk pathogens before they emerge, optimizing vaccine design with an eye to evolution, behaviour and epidemiology, and minimizing the risk of unintended consequences.
\end{abstract}

$\mathrm{n}$ the last few decades, viral zoonoses have become part of the global mindset: Ebola, H1N1 influenza, SARS, COVID-19, MERS and Nipah are all infectious diseases that developed into epidemics, sometimes contained and sometimes not. There are also many viral zoonoses that routinely or sporadically spillover into human or livestock populations but have so far not led to major self-propagating epidemics: rabies, rodent-borne arenaviruses (for example, Lassa, Junin, Machupo and Lujo), and hantaviruses (for example, Sin Nombre virus) ${ }^{1-5}$. For most of these pathogens, human and livestock vaccines do not yet exist. The result is a chronic and substantial burden on human health and well-being for those viruses that spillover regularly (for example, Lassa and rabies) and a reliance on contact tracing and quarantine when sustained transmission within humans becomes established (for example, Ebola and SARS-CoV-2). Although technological advances are continually reducing the time required for vaccine development ${ }^{6-9}$ and beginning to automate the process of contact tracing ${ }^{10}$, the failure to contain the SARS-CoV-2 pandemic illustrates that those methods remain inadequate with more than five million sickened and hundreds of thousands dead only five months after the virus was first detected in Wuhan, China, in late December 2019.

A promising approach for mitigating the public health burden of chronic spillover and reducing the threat of future pandemics is to shift our focus to target high-risk pathogens within their animal reservoirs before viral spillover and/or emergence can occur ${ }^{11}$. Precedent for this approach is long-standing, with wildlife vaccination programmes in Europe and North America substantially reducing the risk of rabies infection for the human population ${ }^{12}$. An additional advantage of proactively vaccinating the animal reservoir rather than the human population itself is that long-term risk reduction through pathogen elimination or eradication becomes possible. For other threats, however, the inaccessibility and rapid turnover of reservoir populations confounds standard vaccination practices to suppress viruses at the source. Advances in genetic engineering now raise the possibility of overcoming these challenges through the use of self-disseminating vaccines capable of transferring from one individual to the next $\mathrm{t}^{13,14}$.
Self-disseminating vaccines have their roots in the Australian effort to create sterilizing vaccines for small mammal control ${ }^{15,16}$, and have also been developed and tested experimentally as a tool for vaccinating rabbits against myxomatosis and rabbit haemorrhagic fever ${ }^{17-19}$. Their obvious advantage, of course, is that for each animal you vaccinate directly, additional animals are vaccinated for 'free' either through behavioural transmission of a conventional vaccine or through the contagious spread of a transmissible vaccine. There are two possible applications for self-disseminating vaccines, one that can be realized now and another that is more aspirational. The immediate application focuses on well-characterized pathogens such as rabies and Lassa virus that regularly spillover into the human population from known animal reservoirs. The aspirational application envisions the possibility of preventing future pandemics by eliminating high-risk zoonotic pathogens from their animal reservoirs before spillover into the human population occurs. Despite their promise, self-disseminating vaccines have not yet been used to reduce the risk of viral spillover into humans. In this Perspective, we begin by reviewing the basic epidemiological theory establishing the feasibility and utility of self-disseminating vaccines. We then outline a road map for addressing remaining technical challenges and design decisions: identifying high-risk pathogens before they emerge, optimizing vaccine design and minimizing the risk of unintended consequences.

\section{Not all self-dissemination is equal}

Mathematical and computational models demonstrate that self-disseminating vaccines reduce the effort required to eliminate human pathogens from their wildlife reservoirs ${ }^{20-22}$. The magnitude of the benefits provided by vaccine self-dissemination, however, depends on the type of self-disseminating vaccine and on elements of vaccine epidemiology, which in turn depend on how the vaccine is created and released. A major distinction among self-disseminating vaccines with considerable epidemiological consequences is whether the vaccine is 'transmissible' and capable of indefinite transmission or is 'transferable' and restricted to a single round of transmission (Fig. 1). In the next sections, we review the basic epidemiological theory quantifying the gains

'Department of Biological Sciences, University of Idaho, Moscow, ID, USA. ${ }^{2}$ Department of Mathematics, University of Idaho, Moscow, ID, USA.

凶e-mail: snuismer@uidaho.edu 
a

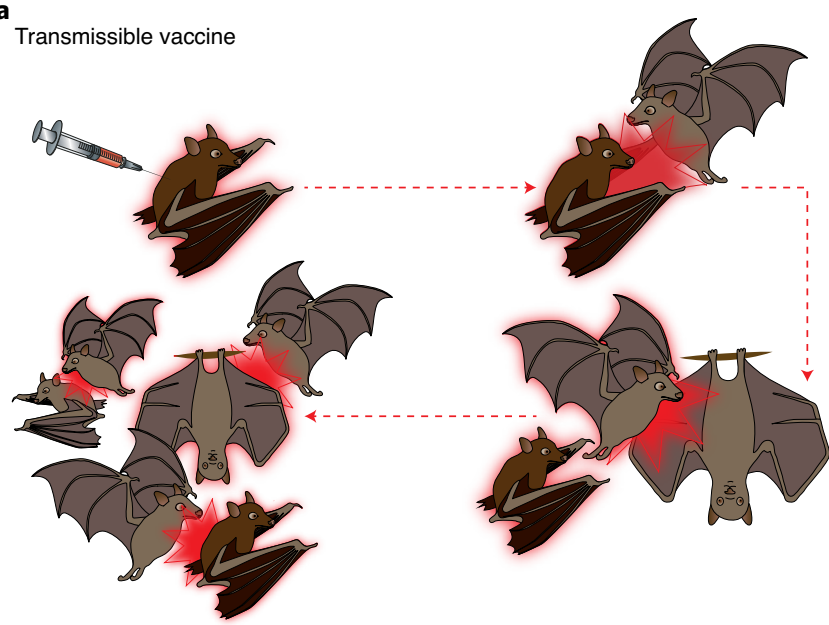

b

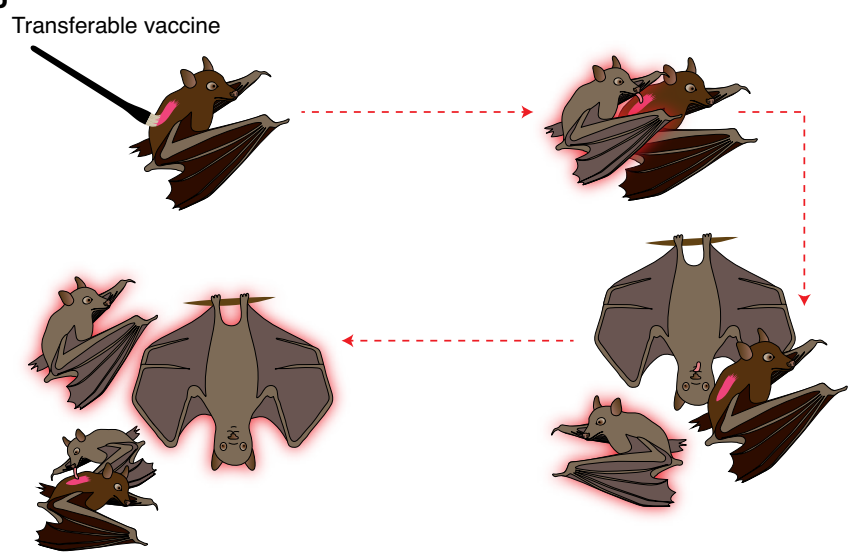

Fig. 1 | Schematic of transmissible and transferable vaccines.

a, A transmissible vaccine is administered directly to one bat via injection. This bat (red outline) is then capable of vaccine transmission. In subsequent time steps (separated by dashed red lines), this initial bat encounters other animals and transmits the vaccine to them infectiously (red bursts). Infectiously vaccinated bats also go on to transmit the vaccine infectiously to others. $\mathbf{b}$, A transferable vaccine is administered directly to a focal bat as a paste to its fur. In subsequent time steps, different bats groom the fur of the focal bat and become vaccinated. The vaccine is not contagious in the usual sense; the focal bat merely serves as a delivery vehicle to the others. Credit: Katy Riendeau

provided by each type of self-disseminating vaccine. These theoretical results use the classical epidemiological concept of a basic reproductive number, $R_{0}$, that quantifies the number of secondary 'vaccine infections' created by the first vaccinated individual in the population.

'Transmissible' vaccines reduce vaccination effort. Transmissible vaccines capable of infectious spread through a reservoir population reduce the vaccination effort required to suppress a target pathogen. With high enough transmission, a transmissible vaccine allows for autonomous pathogen eradication ${ }^{20}$. These benefits of vaccine transmission can be easily quantified in simple models where the reservoir population is assumed to be homogeneous and well-mixed and the vaccine can be continuously introduced into the reservoir population at a rate $\sigma$. In this idealized scenario, the reduction in the vaccination effort required to eliminate the pathogen (relative to a non-transmissible vaccine) is given by the factor $\rho$ :

$$
\rho=\frac{R_{0, \mathrm{~V}}}{R_{0, \mathrm{P}}}
$$

where $R_{0, \mathrm{~V}}$ and $R_{0, \mathrm{P}}$ measure the average number of new infections produced by an infectious individual introduced into an entirely susceptible population (for the vaccine and pathogen, respectively $)^{20}$. This result establishes guiding principles for the engineering and use of transmissible vaccines that achieve a desired impact on the pathogen. If the sole concern is rapid pathogen extirpation, transmissible vaccines with greater $R_{0, \mathrm{~V}}$ values will always be preferable, and autonomous eradication will require a vaccine with an $R_{0, \mathrm{~V}}$ greater than that of the target pathogen. This requirement will be particularly important for pathogens with reservoir species that are difficult to vaccinate directly, or pathogens that circulate in regions lacking the resources and infrastructure to implement regular vaccine introduction. In contrast, if regular introduction of the vaccine into the reservoir population is feasible and concerns about vaccine safety predominate, pathogen eradication can be facilitated using a vaccine with an $R_{0, \mathrm{~V}}<1$ that will self-extinguish once introduction ceases. In both cases, vaccine self-dissemination facilitates pathogen control and/or elimination, although these benefits can obviously be magnified manifold for highly transmissible vaccines with $R_{0, \mathrm{~V}}$ values well above 1 .

One-step, 'transferable' vaccines reduce vaccination effort despite being dead ends. It might seem that a vaccine which transfers only a single step is at a strong disadvantage relative to one that transmits indefinitely. There is indeed a disadvantage of limited transmission, but not necessarily much ${ }^{23}$. For instance, in a homogeneous and well-mixed reservoir population, a transferable vaccine reduces the vaccination effort required to eradicate a target pathogen by a proportion $\rho$ :

$$
\rho=\frac{R_{0, \mathrm{~V}}}{\left(R_{0, \mathrm{~V}}+R_{0, \mathrm{P}}\right)}
$$

Comparing this result (equation (2)) with our earlier result for a transmissible vaccine (equation (1)) shows that, all else being equal, a single-step transferable vaccine will always perform worse than a fully transmissible vaccine. However, comparing equations (1) and (2) and their respective panels in Fig. 2 shows that this difference is negligible for weakly self-disseminating vaccines. Biologically, this quasi-equivalence occurs because even fully transmissible vaccines with low $R_{0, \mathrm{~V}}$ produce only short chains of transmission, reducing their advantage over single-step transferable vaccines. As vaccine $R_{0, \mathrm{~V}}$ increases, however, the advantage of a transmissible vaccine becomes more appreciable. Most importantly, a transmissible vaccine will automatically displace the pathogen whenever the vaccine has the higher $R_{0}$, whereas a transferable vaccine has no such possibility - it must be applied continually up to the point that the pathogen is extinguished.

\section{High-risk pathogens and their reservoirs}

To design and use a self-disseminating vaccine effectively, we minimally require a modest understanding of pathogen epidemiology and the distribution and ecology of its zoonotic reservoir(s). For some important human pathogens, this is a relatively straightforward task because the reservoir species and pathogen are well known. For instance, rabies, Lassa and Sin Nombre viruses are well characterized and their reservoir species relatively well understood ${ }^{12,24-27}$. Rabies virus, in particular, makes a compelling target for development of a self-disseminating vaccine because effective wildlife vaccines already exist-the only remaining hurdle is achieving self-dissemination.

Still, even for a virus as well studied as rabies, challenges must be overcome. Perhaps the most important is the circulation of the 

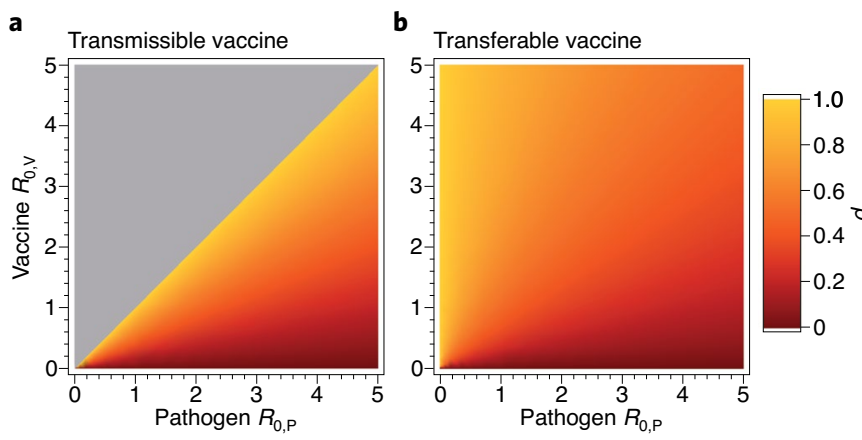

Fig. 2 | The reduction in vaccination effort $(\rho)$ provided by vaccine self-dissemination for pathogens and vaccines with differing $\boldsymbol{R}_{0}$. $\mathbf{a}$, The reduction in effort provided by a transmissible vaccine. $\mathbf{b}$, The reduction in effort afforded by a transferable vaccine. The grey area in $\mathbf{a}$ shows the region of parameter space in which a single introduction of the transmissible vaccine is sufficient to eliminate the pathogen (autonomous pathogen eradication).

virus within multiple wildlife reservoirs ${ }^{28}$, each of which contributes to the global persistence of rabies ${ }^{29}$. Fortunately, because rabies virus generally persists in species-specific transmission cycles, spillover risk from any particular reservoir can be reduced using a single self-disseminating vaccine. Global eradication of rabies would be more complex, of course, and require development of multiple self-disseminating vaccines, each targeting a different reservoir species. Lassa virus also makes an obvious target for development of self-disseminating vaccines because the virus itself is well characterized, and we have a relatively good understanding of the primary reservoir and its ecology ${ }^{24,30,31}$. Here too, however, multiple rodent species have been shown to harbour the virus ${ }^{25}$ and we do not yet know what fraction of spillover into the human population can be attributed to the primary reservoir, Mastomys natalensis, and what fraction to the remaining secondary reservoirs. Other human pathogens that make more challenging candidates for the application of self-disseminating vaccines include Marburg, Hendra and some well-studied coronaviruses ${ }^{4,32-35}$.

Beyond application to these relatively well-studied viruses, self-disseminating vaccines could also be used to pre-empt spillover and emergence of novel pathogens. The challenge, of course, is predicting which of the myriad viruses circulating within wild animal populations represents imminent threats of emergence into the human population. Developing this capacity will require investment and expansion of global surveillance efforts within wild animals ${ }^{11,36}$. For instance, the US Agency for International Development (USAID) PREDICT programme has identified more than 949 unique viruses, including the discovery of a novel Ebolavirus, and has improved our understanding of viral distributions and reservoir species ${ }^{34,35,37-39}$. Surveillance alone is not sufficient, however, and needs to be coupled with new methods that capitalize on advances in genomics, phylogenetics and machine learning to predict which novel viruses represent imminent threats of emergence, as well as their primary animal reservoirs ${ }^{40-46}$. Despite these advances in viral surveillance and risk forecasting, it remains unclear to what extent we will ever be able to reliably anticipate which novel pathogens are likely to emerge in the near future. Thus, at least for the near term, self-disseminating vaccines are likely to have the greatest impact on human and animal health when applied to well-known viruses with an established history of spillover and emergence into the human population.

\section{Strategies for implementation}

Advances in genetic engineering have enabled the development of self-disseminating vaccines, but success will also rest on optimizing implementation with an eye toward ecology, evolution and epidemiological constraints. In the following, we explain some of the more important considerations that are likely to affect the first generation of self-disseminating vaccines.

Tailoring to host biology. For transferable (one-step) vaccines, the primary challenge is identifying behavioural patterns of reservoir species that can be used to disseminate the vaccine with a high one-step $R_{0, \mathrm{~V}}$. The best studied of these deliveries is allogrooming in bats, where individuals within a colony groom each other and provide an opportunity for the widespread oral transmission of vaccines delivered to the bat's fur. Using topical application to individual bats of rhodamine $b$, a biomarker that causes fluorescence in hair follicles after ingestion, Bakker et al. ${ }^{47}$ demonstrated that each direct application led to ingestion by between 1.45 and 2.11 additional bats. Another behaviour that might provide an effective avenue for single-step vaccine transfer is nursing in mammals, either through topical application or development of vaccine baits that can be excreted in milk and ingested by offspring or disseminated more broadly through allosuckling ${ }^{48}$. A secondary challenge in designing effective transferable vaccines is optimizing the vaccine itself so that it can be effectively passed to other individuals. Thus, issues of vaccine concentration, the matrix in which it is embedded and even the anatomical sites of delivery will need to be worked out ${ }^{49}$. There may also be strong seasonality in some systems that can be used to tune the timing of vaccine delivery to magnify impact on population-level immunity ${ }^{50}$.

Success of transmissible vaccines will also benefit from delivery to individuals with specific behaviours. Choice of who to directly vaccinate will be most important in planning the initial introduction of the vaccine and may also influence the rate and extent of spread through the reservoir population. For instance, timing the introduction of the transmissible vaccine to coincide with seasonal birth pulses in the reservoir species may increase the likelihood of vaccine establishment and spread ${ }^{50}$. Vaccine transmission may also differ among classes of individuals (for example, be higher from aggressive males or new mothers), so choice of which individuals to initially vaccinate may have a large influence on the success of vaccine introductions ${ }^{51}$. Spatial structure of the reservoir, too, is likely to influence the spread of a transmissible vaccine and to be an important consideration in planning the number and location of initial introductions ${ }^{21}$. In addition to these impacts on initial establishment, reservoir species biology may influence the long-term rate of vaccine spread through the population, creating challenges and opportunities for transmissible vaccine design, particularly the choice of viral vectors.

Attenuated versus recombinant vector vaccines. Two types of live vaccine design potentially suitable for self-dissemination are attenuated and recombinant vector vaccines (Fig. 3). Attenuated vaccines are wild-type viruses modified to avoid pathogenesis, usually by reducing viral growth rate. Recombinant vector vaccines are developed by inserting immunogenic genes from the target pathogen into a competent but innocuous viral vector. For single-step transferable vaccines, the decision between these two types of vaccine designs may be of little importance because the ability to transmit infectiously from host to host does not need to be maintained. For transmissible vaccines, however, the choice between attenuated and recombinant designs may determine how well the vaccine is able to self-disseminate.

Effective transmissible vaccines developed using attenuation must maintain considerable levels of transmission while producing minimal disease. Yet, evolutionary theory and observations from many attenuated vaccines suggest that reduced disease (or virulence) and decreased rates of transmission can go hand in hand ${ }^{52}$. Thus, developing safe but highly transmissible attenuated vaccines 


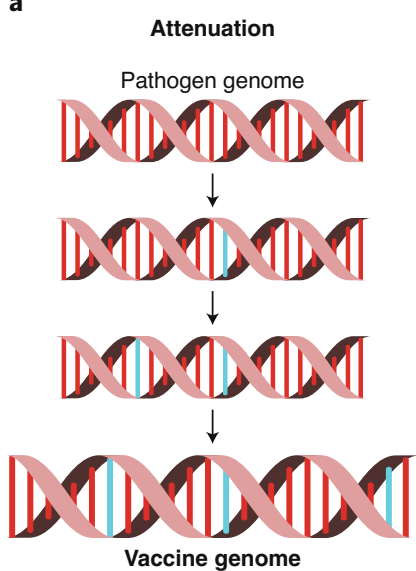

Oral polio vaccine (OPV) b

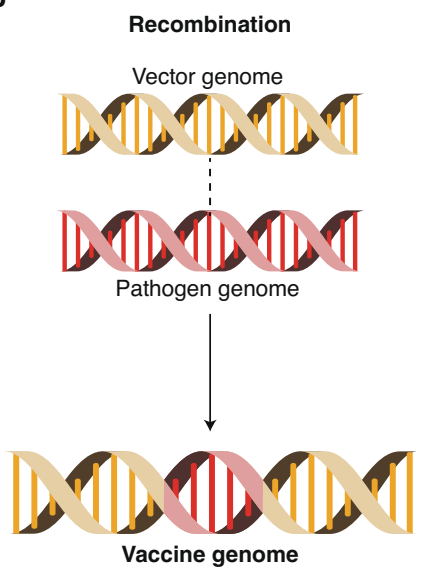

Rabbit haemorrhagic disease vaccine
Fig. 3 | Two methods for building transmissible vaccines. a, Attenuation is used to reduce the virulence of the wild-type pathogen. Attenuation is shown here as the gradual accumulation of point mutations (blue). b. Recombination is used to insert an immunogenic region of the pathogen genome (red) into the genome of an innocuous but transmissible viral vector (yellow). Credit: Katy Riendeau

may be challenging. An additional problem confronting attenuated transmissible vaccines is the possibility of evolution returning the vaccine to its wild-type and pathogenic state. For instance, we now know that oral polio vaccine (OPV) is transmissible and readily evolves back to wild-type virulence ${ }^{53}$. Although new methods of attenuation greatly limit evolutionary reversion ${ }^{54,55}$, an attenuated vaccine whose $R_{0}$ exceeds 1 will always pose an ongoing threat of reversion if the wild-type pathogen has been extinguished, enabling the attenuated virus to persist indefinitely. For this reason, attenuated transmissible vaccines are unlikely to ever be suitable tools for eliminating human pathogens from their wildlife reservoirs. They may, however, be safe and effective tools for controlling pathogens that exclusively infect wild or agricultural animal populations and pose no risk of infection for humans, as long as the benefits gained from vaccination outweigh the costs of reversion to wild-type virulence at the population level ${ }^{20}$.

Recombinant vector vaccines avoid both problems of attenuated transmissible vaccines. Transmission should depend primarily on the vector, making it possible to develop transmissible vaccines with an $R_{0}$ exceeding that of the target pathogen while maintaining the avirulent phenotype of the vector. It may also be possible to enhance vector $R_{0}$ by selecting highly transmissible vector strains or even adapting the vector for rapid transmission using serial passages through captive reservoir populations. Consequently, recombinant vaccines are a priori the most promising approach for a transmissible vaccine. Furthermore, because selection favouring increased transmission is likely to favour mutations resulting in the loss or downregulation of the immunogenic insert, we expect the most likely outcome of evolution to be a return to the innocuous viral vector. Thus, evolution may reduce the effectiveness of recombinant vaccines, but is unlikely to result in increased virulence or pathogenicity ${ }^{56}$.

Evading prevailing immunity. Spread of transferable and transmissible vaccines is impeded by pre-existing immunity to the vaccine: pre-existing immunity effectively reduces vaccine $R_{0}$ and thus requires correspondingly greater effort (for example, equations (1) and (2)). For transmissible vaccines using a recombinant vector design, prevailing host immunity to either vector or pathogen will slow vaccine spread ${ }^{22}$. Host immunity may thus strongly influence choice of a vector: viruses in the genus Cytomegalovirus (CMV) and their relatives in the Betaherpesvirinae are considered promising candidates because they have a demonstrated propensity for superinfection and apparent lack of protective immunity ${ }^{14}$. Alternative approaches to reducing the burden of pre-existing immunity to the vector include using a foreign vector that does not naturally infect the reservoir species or using rare strains of an endemic vector for which only limited immunity exists ${ }^{9}$. Using a foreign vector does, however, carry substantial risks unlikely to be justifiable in most cases.

\section{Proof of concept}

Although our understanding of self-disseminating vaccines and their promise remains largely theoretical, empirical studies have demonstrated that both transmissible and transferable vaccines can be developed. The best-studied transmissible vaccine was created using a naturally attenuated myxoma virus that was engineered as a recombinant vector vaccine against rabbit haemorrhagic disease $^{17-19}$. Field trials of this transmissible vaccine were conducted by releasing 76 directly vaccinated animals and 71 unvaccinated sentinel animals onto the small Isla del Aire, off the coast of Spain ${ }^{18}$. After 32 days, 25 sentinel animals were recaptured; $56 \%$ had seroconverted due to indirect vaccination. Back of the envelope calculations show that these values suggest an $R_{0}$ for the vaccine of between 1.39 and 2.11 , depending on what is assumed about the recovery rate from vaccine infection. This study demonstrates the feasibility of a transmissible vaccine and also illustrates additional possibilities for vaccine design that use attenuated viruses as the vector.

As noted above, the potential feasibility of a transferable vaccine was demonstrated for vampire bats using ingestible dyes placed on the fur of index bats ${ }^{47}$. Their results suggest that topical application of a rabies vaccine would yield a transferable vaccine with an effective $R_{0}$ of 1.45-2.11. From our equation (2), this degree of self-dissemination would reduce the vaccination effort required for rabies elimination by up to $51.3 \%$, assuming rabies has an $R_{0} \leq 2.0$, as was suggested for this system. Combined with earlier work demonstrating the feasibility of developing a topically applied recombinant vector vaccine against rabies ${ }^{49}$, these results demonstrate that a transferable vaccine is within our immediate reach.

\section{Minimizing deleterious and unintended consequences}

Self-disseminating vaccines may well come with risks, some of which are already clear and others of which we may not yet be aware. For transferable vaccines, at least, the risks are well understood and no greater than those associated with current vaccination campaigns that rely on the widespread distribution of vaccine-laced baits. For transmissible vaccines, the risks may be greater because their sustained replication and transmission creates substantial opportunities for evolution. A case in point is the evolution and escape of the live polio vaccine, which now circulates and causes disease $e^{53,57}$. The risk can be reduced, however, by using recombinant vector vaccines and perhaps, if using attenuated vaccines, by using new methods to engineer attenuation ${ }^{54,55}$.

Although recombinant vaccine evolution is not expected to be harmful, an additional layer of safety may be achieved by engineering vaccines to self-extinguish, losing the antigenic insert on a schedule ${ }^{56}$. Even so, it would be naive to believe that recombinant vector vaccines are without risk. For instance, using a novel vector that is not circulating in the animal reservoir has the advantage of avoiding prevailing immunity but runs the risk of unknown evolution and virulence upon release. Further, there is at least some possibility that the immunogenic insert could be co-opted by the viral vector to expand its ecological niche by allowing access to new tissues or even hosts.

From this understanding, there are actions that can be taken to reduce unanticipated consequences of transmissible vaccines: 
(1) use recombinant vector rather than attenuated vaccines (or at least avoid attenuated vaccines derived from the pathogen); (2) use species-specific vectors; (3) engineer self-regulatory mechanisms; and (4) use transmissible vaccines whose $R_{0}$ assures eventual loss from the population. It will often be impractical to adhere to all of these recommendations, and caution may give way to expedience if the consequences of pathogen spillover are great. Following these basic design principles as guidelines as closely as possible will, however, minimize the possibility of unanticipated consequences.

Finally, the safe and effective use of transmissible viral vaccines will require carefully staged development with effectiveness and risk evaluated at multiple checkpoints. For instance, once a candidate transmissible vaccine has been developed, its effectiveness, transmission and evolution should be studied using captive animal populations, also testing the potential for transmission into closely related reservoir species. Results from these initial studies with captive animals can be used to refine mathematical models predicting how well the vaccine is likely to work, in turn evaluating the potential gains for human and animal health. The obvious next step is to perform releases within carefully isolated populations in semi-natural enclosures or on small islands. Precedent for this approach was established during the first field trials of a transmissible vaccine targeting myxoma and rabbit haemorrhagic fever ${ }^{18}$. Here, too, effectiveness, transmission and evolution should be studied and used to further refine models predicting likely gains that could be realized by release into natural populations. Throughout development and testing, regulatory agencies and stakeholders should be involved so that concerns can be addressed during controlled experiments, evaluating the road to release within natural populations.

\section{Conclusions}

Self-disseminating vaccines represent an opportunity to completely change how we approach emerging infectious disease. Rather than responding to outbreaks or epidemics, self-disseminating vaccines make it possible to prevent them in the first place. However, there is much we do not yet know about the performance of self-disseminating vaccines under real-world conditions. A logical first step is to begin developing, testing and deploying single-step transferable vaccines. Their risks are minimal and their benefits demonstrable. On the downside, transferable vaccines are likely to only modestly transform our ability to eliminate viral spillover and may be restricted to a narrow range of systems with particular behaviours, such as allogrooming. Transmissible vaccines, in contrast, entail greater risk but also have a much greater scope for eliminating hard to reach pathogens from their animal reservoirs. Thus, the logical next step is to begin developing and testing transmissible vaccines for a handful of well-understood systems where risks can be well managed as we develop a better understanding of the evolutionary epidemiology of these new tools. Although the financial investment required to develop and test the first generation of transmissible vaccines is likely to be substantial, it is inconsequential when compared to the cost of viral spillover-more than US\$3.6 billion for the response to the West African Ebola epidemic of 2014-2016, US $\$ 40$ billion for the SARS outbreak of 2003, and US $\$ 8$ billion per year for canine rabies alone $e^{58-60}$.

Received: 10 April 2020; Accepted: 22 June 2020;

Published online: 27 July 2020

\section{References}

1. Redding, D. W., Moses, L. M., Cunningham, A. A., Wood, J. \& Jones, K. E. Environmental-mechanistic modelling of the impact of global change on human zoonotic disease emergence: a case study of Lassa fever. Methods Ecol. Evol. 7, 646-655 (2016).

2. McCormick, J. B. \& Fisher-Hoch, S. P. in Arenaviruses I: The Epidemiology, Molecular and Cell Biology of Arenaviruses - Current Topics in Microbiology and Immunology Vol. 262 (ed. Oldstone, M. B. A.) 75-109 (Springer, 2002).
3. Jonsson, C. B., Figueiredo, L. T. M. \& Vapalahti, O. A global perspective on hantavirus ecology, epidemiology, and disease. Clin. Microbiol. Rev. 23, 412-441 (2010).

4. Edson, D. et al. Routes of Hendra virus excretion in naturally-infected flying-foxes: implications for viral transmission and spillover risk. PLoS ONE 10, e0140670 (2015).

5. Luby, S. P., Gurley, E. S. \& Jahangir Hossain, M. Transmission of human infection with Nipah virus. Clin. Infect. Dis. 49, 1743-1748 (2009).

6. Georgiou, G. et al. Display of heterologous proteins on the surface of microorganisms: from the screening of combinatorial libraries to live recombinant vaccines. Nat. Biotechnol. 15, 29-34 (1997).

7. Leitner, W. W., Ying, H. \& Restifo, N. P. DNA and RNA-based vaccines: principles, progress and prospects. Vaccine 18, 765-777 (1999).

8. Pardi, N., Hogan, M. J., Porter, F. W. \& Weissman, D. mRNA vaccines - a new era in vaccinology. Nat. Rev. Drug Discov. 17, 261-279 (2018).

9. Rollier, C. S., Reyes-Sandoval, A., Cottingham, M. G., Ewer, K. \& Hill, A. V. S. Viral vectors as vaccine platforms: deployment in sight. Curr. Opin. Immunol. 23, 377-382 (2011).

10. Ferretti, L. et al. Quantifying SARS-CoV-2 transmission suggests epidemic control with digital contact tracing. Science 368, eabb6936 (2020).

11. Morse, S. S. et al. Prediction and prevention of the next pandemic zoonosis. Lancet 380, 1956-1965 (2012).

12. Rupprecht, C. E., Hanlon, C. A. \& Slate, D. in Control of Infectious Animal Diseases by Vaccination - Developments in Biologicals Vol. 119 (eds Schudel, A. \& Lombard, M.) 173-184 (Karger, 2004).

13. Bull, J. J., Smithson, M. W. \& Nuismer, S. L. Transmissible viral vaccines. Trends Microbiol. 26, 6-15 (2018).

14. Murphy, A. A., Redwood, A. J. \& Jarvis, M. A. Self-disseminating vaccines for emerging infectious diseases. Expert Rev. Vaccines 15, 31-39 (2016).

15. Shellam, G. R. The potential of murine cytomegalovirus as a viral vector for immunocontraception. Reprod. Fertil. Dev. 6, 401-409 (1994)

16. Tyndale-Biscoe, C. H. Virus-vectored immunocontraception of feral mammals. Reprod. Fertil. Dev. 6, 281-287 (1994).

17. Barcena, J. et al. Horizontal transmissible protection against myxomatosis and rabbit hemorrhagic disease by using a recombinant myxoma virus. J. Virol. 74, 1114-1123 (2000).

18. Torres, J. M. et al. First field trial of a transmissible recombinant vaccine against myxomatosis and rabbit hemorrhagic disease. Vaccine 19 4536-4543 (2001).

19. Angulo, E. \& Barcena, J. Towards a unique and transmissible vaccine against myxomatosis and rabbit haemorrhagic disease for rabbit populations. Wildl. Res. 34, 567-577 (2007)

20. Nuismer, S. L. et al. Eradicating infectious disease using weakly transmissible vaccines. Proc. R. Soc. B 283, 20161903 (2016).

21. Basinski, A. J., Nuismer, S. L. \& Remien, C. H. A little goes a long way: weak vaccine transmission facilitates oral vaccination campaigns against zoonotic pathogens. PLoS Negl. Trop. Dis. 13, e0007251 (2019).

22. Basinski, A. J. et al. Evaluating the promise of recombinant transmissible vaccines. Vaccine 36, 675-682 (2018).

23. Smithson, M. W., Basinki, A. J., Nuismer, S. L. \& Bull, J. J. Transmissible vaccines whose dissemination rates vary through time, with applications to wildlife. Vaccine 37, 1153-1159 (2019).

24. Lecompte, E. et al. Mastomys natalensis and Lassa fever, West Africa. Emerg. Infect. Dis. 12, 1971-1974 (2006).

25. Olayemi, A. et al. New hosts of the Lassa virus. Sci. Rep. 6, 25280 (2016).

26. Douglass, R. J. et al. Longitudinal studies of Sin Nombre virus in deer mouse-dominated ecosystems of Montana. Am. J. Trop. Med. Hyg. 65, 33-41 (2001).

27. Luis, A. D., Douglass, R. J., Mills, J. N. \& Bjornstad, O. N. The effect of seasonality, density and climate on the population dynamics of Montana deer mice, important reservoir hosts for Sin Nombre hantavirus. J. Anim. Ecol. 79, 462-470 (2010)

28. Viana, M. et al. Assembling evidence for identifying reservoirs of infection. Trends Ecol. Evol. 29, 270-279 (2014).

29. Fenton, A., Streicker, D. G., Petchey, O. L. \& Pedersen, A. B. Are all hosts created equal? Partitioning host species contributions to parasite persistence in multihost communities. Am. Nat. 186, 610-622 (2015).

30. Fichet-Calvet, E. et al. Fluctuation of abundance and Lassa virus prevalence in Mastomys natalensis in Guinea, West Africa. Vector-Borne Zoonotic Dis. 7, 119-128 (2007).

31. Marien, J. et al. Evaluation of rodent control to fight Lassa fever based on field data and mathematical modelling. Emerg. Microbes Infect. 8, 640-649 (2019).

32. Towner, J. S. et al. Marburg virus infection detected in a common african bat. PLoS ONE 2, e764 (2007).

33. Nziza, J. et al. Coronaviruses detected in bats in close contact with humans in Rwanda. EcoHealth 17, 152-159 (2020).

34. Anthony, S. J. et al. Further evidence for bats as the evolutionary source of Middle East respiratory syndrome coronavirus. Mbio 8, e00373-17 (2017). 
35. Ge, X.-Y. et al. Isolation and characterization of a bat SARS-like coronavirus that uses the ACE2 receptor. Nature 503, 535-538 (2013).

36. Bird, B. H. \& Mazet, J. A. K. Detection of emerging zoonotic pathogens: an integrated one health approach. Annu. Rev. Anim. Biosci. 6, 121-139 (2018).

37. Goldstein, T. et al. The discovery of Bombali virus adds further support for bats as hosts of ebolaviruses. Nat. Microbiol. 3, 1084-1089 (2018).

38. Pernet, O. et al. Evidence for henipavirus spillover into human populations in Africa. Nat. Commun. 5, 5342 (2014).

39. Grard, G. et al. A novel rhabdovirus associated with acute hemorrhagic fever in Central Africa. PLoS Pathog. 8, e1002924 (2012).

40. Han, B. A. \& Drake, J. M. Future directions in analytics for infectious disease intelligence. EMBO Rep. 17, 785-789 (2016).

41. Han, B. A., Schmidt, J. P., Bowden, S. E. \& Drake, J. M. Rodent reservoirs of future zoonotic diseases. Proc. Natl Acad. Sci. USA 112, 7039-7044 (2015).

42. Han, B. A. et al. Undiscovered bat hosts of filoviruses. PLoS Negl. Trop. Dis. 10, e0004815 (2016)

43. Guth, S., Visher, E., Boots, M. \& Brook, C. E. Host phylogenetic distance drives trends in virus virulence and transmissibility across the animal-human interface. Philos. Trans. R. Soc. B 374, 20190296 (2019).

44. Olival, K. J. et al. Host and viral traits predict zoonotic spillover from mammals. Nature 546, 646-650 (2017)

45. Pepin, K. M., Lass, S., Pulliam, J. R. C., Read, A. F. \& Lloyd-Smith, J. O. Identifying genetic markers of adaptation for surveillance of viral host jumps. Nat. Rev. Microbiol. 8, 802-813 (2010).

46. Babayan, S. A., Orton, R. J. \& Streicker, D. G. Predicting reservoir hosts and arthropod vectors from evolutionary signatures in RNA virus genomes. Science 362, 577-580 (2018).

47. Bakker, K. M. et al. Fluorescent biomarkers demonstrate prospects for spreadable vaccines to control disease transmission in wild bats. Nat. Ecol. Evol. 3, 1697-1704 (2019).

48. Garnier, R., Gandon, S., Chaval, Y., Charbonnel, N. \& Boulinier, T. Evidence of cross-transfer of maternal antibodies through allosuckling in a mammal: potential importance for behavioral ecology. Mamm. Biol. 78, 361-364 (2013).

49. Stading, B. et al. Protection of bats (Eptesicus fuscus) against rabies following topical or oronasal exposure to a recombinant raccoon poxvirus vaccine. PLoS Negl. Trop. Dis. 11, e0005958 (2017).

50. Schreiner, C. L., Nuismer, S. L. \& Basinski, A. J. When to vaccinate a fluctuating wildlife population: is timing everything? J. Appl. Ecol. 57, 307-319 (2020)
51. Varrelman, T. J., Basinski, A. J., Remien, C. H. \& Nuismer, S. L. Transmissible vaccines in heterogeneous populations: implications for vaccine design. One Health 7, 100084 (2019).

52. Alizon, S., Hurford, A., Mideo, N. \& Van Baalen, M. Virulence evolution and the trade-off hypothesis: history, current state of affairs and the future. J. Evol. Biol. 22, 245-259 (2009).

53. Kew, O. M., Sutter, R. W., de Gourville, E. M., Dowdle, W. R. \& Pallansch, M. A. Vaccine-derived polioviruses and the endgame strategy for global polio eradication. Annu. Rev. Microbiol. 59, 587-635 (2005).

54. Bull, J. J. Evolutionary reversion of live viral vaccines: can genetic engineering subdue it? Virus Evol. 1, vev005 (2015).

55. Lauring, A. S., Jones, J. O. \& Andino, R. Rationalizing the development of live attenuated virus vaccines. Nat. Biotechnol. 28, 573-579 (2010).

56. Nuismer, S. L., Basinski, A. \& Bull, J. J. Evolution and containment of transmissible recombinant vector vaccines. Evol. Appl. 12, 1595-1609 (2019).

57. Kew, O. M. et al. Circulating vaccine-derived polioviruses: current state of knowledge. Bull. World Health Organ. 82, 16-23 (2004).

58. Hampson, $\mathrm{K}$. et al. Estimating the global burden of endemic canine rabies. PLoS Negl. Trop. Dis. 9, e0003709 (2015).

59. Cost of the Ebola Epidemic (US Centers for Disease Control and Prevention, 2020); https://go.nature.com/38iF7cg

60. Forum on Microbial Threats Learning from SARS: Preparing for the Next Disease Outbreak: Workshop Summary (National Academies Press, 2004).

\section{Acknowledgements}

We thank K. Riendeau for developing Figs. 1 and 3. S.L.N. and J.J.B. were supported by National Institutes of Health (NIH) grant no. R01GM122079.

\section{Author contributions}

S.L.N. and J.J.B. conceived of this Perspective and contributed to writing and revision.

\section{Competing interests}

The authors declare no competing interests.

\section{Additional information}

Correspondence should be addressed to S.L.N.

Reprints and permissions information is available at www.nature.com/reprints.

Publisher's note Springer Nature remains neutral with regard to jurisdictional claims in published maps and institutional affiliations.

(C) Springer Nature Limited 2020 